\title{
CONSISTENCY, VERIFICATION, AND VALIDATION OF TURBULENCE MODELS FOR REYNOLDS-AVERAGED NAVIER-STOKES APPLICATIONS
}

\author{
C. L. Rumsey \\ NASA Langley Research Center, Hampton, VA 23681-2199
}

\begin{abstract}
In current practice, it is often difficult to draw firm conclusions about turbulence model accuracy when performing multi-code CFD studies ostensibly using the same model because of inconsistencies in model formulation or implementation in different codes. This paper describes an effort to improve the consistency, verification, and validation of turbulence models within the aerospace community through a website database of verification and validation cases. Some of the variants of two widely-used turbulence models are described, and two independent computer codes (one structured and one unstructured) are used in conjunction with two specific versions of these models to demonstrate consistency with grid refinement for several representative problems. Naming conventions, implementation consistency, and thorough grid resolution studies are key factors necessary for success.
\end{abstract}

\section{Introduction}

Computational fluid dynamics (CFD) codes that solve the Reynolds-averaged Navier-Stokes (RANS) equations are applied regularly at government laboratories, universities, and companies throughout the world to predict flow fields in and around complex configurations. A major component of most RANS applications is the turbulence model, which attempts to model the effects of turbulent fluctuations on the mean flow. Many types of turbulence models exist, ranging from algebraic models through linear one- and two-equation and all the way to nonlinear seven-equation full Reynolds-stress. One thing that all models have in common is that they are imperfect: they are unable to reproduce all features of all classes of flows. Unfortunately, it is often difficult to isolate whether the cause of any particular failure of a given code to match an experiment is due to the turbulence model itself, or some other reason (e.g., numerical errors, boundary conditions, geometry).

One disturbing issue that has surfaced over the years is the fact that different codes that use ostensibly the same turbulence model often do not approach the same result for the same case as the grid is refined (see, e.g., Vassberg et al.[1]). Beside inadequately refined grids, two of the contributing causes to this state of affairs is that different codes either knowingly or unknowingly use different versions of a turbulence model, or that a particular implementation has not been thoroughly verified. It can sometimes be difficult to implement turbulence models from the open literature because of poor reporting practices [2]. Verification of turbulence models can also be problematic. One way is through the method of manufactured solutions (MMS), but only limited solutions are available for turbulence (see, e.g., Eca et al.[3]). Demonstrating consistency using the same model in different codes can provide some level of assurance [4], but optimally the testing should be conducted by independent groups [5] to minimize the possibility of repeating the same mistakes.

In an effort to help improve the consistency, verification, and validation of turbulence models within the aerospace community, NASA has recently established a website to provide a central location where widely-used RANS turbulence models are described, grids and cases are provided, and detailed results are given (http://turbmodels.larc.nasa.gov, cited 19 May 2009). As it grows, this site should serve as a resource to the CFD community for verifying turbulence model implementations. This capability is made possible by providing simple test cases and grids, along with sample results (including complete grid convergence studies) from one or more previously-verified codes. Furthermore, by listing accepted versions of the turbulence models as well as published variants, this site establishes naming conventions in order to help avoid confusion when comparing results from different codes.

The above-mentioned website is also a part of a larger effort, involving a discussion group of the American Institute of Aeronautics and Astronautics (AIAA), to establish a database and standards for turbulence modeling assessment. A similar goal was initially outlined at a turbulence modeling workshop

This material is declared a work of the U.S. Government and is not subject to copyright protection in the United States.2009 
held in 2001, as described in Rubinstein et al.[6]. The current paper primarily describes the verification part of the effort, and stresses the importance of consistency in implementation as well as conducting thorough grid convergence studies.

\section{Examples of Turbulence Model Variants}

Even among the widely-used one-equation Spalart-Allmaras (SA) [7] and two-equation Menter shear stress transport (SST) [8] $k$ - $\omega$ models, several variants exits. Sometimes, these variations are glossed over or ignored when reporting results. This can result in misunderstanding and/or misinterpretation of trends. The current effort seeks to fully document and label many turbulence model variants, thus establishing a basis from which future identification and cross-correlation of models and their results will be possible. This basis is a key component in the effort to achieve consistency between different codes.

The one-equation SA model is written in terms of the turbulence quantity $\tilde{\nu}$ :

$$
\begin{aligned}
& \frac{\partial \tilde{\nu}}{\partial t}+u_{j} \frac{\partial \tilde{\nu}}{\partial x_{j}}=C_{b 1}\left(1-f_{t 2}\right) \tilde{S} \tilde{\nu}-\left[C_{w 1} f_{w}-\frac{C_{b 1}}{\kappa^{2}} f_{t 2}\right]\left(\frac{\tilde{\nu}}{d}\right)^{2} \\
& +\frac{1}{\sigma}\left[\frac{\partial}{\partial x_{j}}\left((\nu+\tilde{\nu}) \frac{\partial \tilde{\nu}}{\partial x_{j}}\right)+C_{b 2} \frac{\partial \tilde{\nu}}{\partial x_{i}} \frac{\partial \tilde{\nu}}{\partial x_{i}}\right]
\end{aligned}
$$

where a description of each of the terms is not given here, but can be found in the original reference [7]. The quantity $\tilde{\nu}$ is related to the eddy viscosity by:

$$
\mu_{t}=\rho \tilde{\nu} \frac{\left(\frac{\tilde{\nu}}{\nu}\right)^{3}}{\left(\frac{\tilde{\nu}}{\nu}\right)^{3}+c_{v 1}^{3}}
$$

where $\nu$ is the molecular kinematic viscosity and $c_{v 1}=7.1$. The so-called SA-la version of this model included an additional trip term, but by common practice most codes do not include it. The recommended far field boundary condition $[9,10]$ for Eq. (1) is in the range of $3 \nu_{\infty} \leq \tilde{\nu}_{\text {farfield }} \leq 5 \nu_{\infty}$, and at the body $\tilde{\nu}_{w a l l}=0$. For all results reported here, $\tilde{\nu}_{\text {farfield }}=3 \nu_{\infty}$.

Some programmers leave out the $f_{t 2}$ term in Eq. (1), since it was originally associated with the use of the trip term. This version of the model, termed here SA-noft2, can be found for example in Eca et al.[3]. Other versions of the Spalart-Allmaras model include SA-RC [11], SA-Catris [12], SA-Edwards [13], and SA-salsa [14].

The two-equation SST model is written in terms of the two turbulence quantities $k$ and $\omega$. The form is:

$$
\begin{aligned}
& \frac{D k}{D t}=\frac{\mathcal{P}}{\rho}-\beta^{*} \omega k+\frac{\partial}{\partial x_{j}}\left[\left(\nu+\sigma_{k} \nu_{t}\right) \frac{\partial k}{\partial x_{j}}\right] \\
& \frac{D \omega}{D t}=\frac{\gamma}{\mu_{t}} \mathcal{P}-\beta \omega^{2}+\frac{\partial}{\partial x_{j}}\left[\left(\nu+\sigma_{\omega} \nu_{t}\right) \frac{\partial \omega}{\partial x_{j}}\right]+2\left(1-F_{1}\right) \frac{\sigma_{\omega 2}}{\omega} \frac{\partial k}{\partial x_{j}} \frac{\partial \omega}{\partial x_{j}}
\end{aligned}
$$

The eddy viscosity is given by:

$$
\mu_{t}=\rho \frac{a_{1} k}{\max \left(a_{1} \omega, \Omega F_{2}\right)}
$$

where $a_{1}=0.31, \Omega$ is the magnitude of vorticity, and $F_{2}$ is a blending function. The production term $\mathcal{P}$ is given by

$$
\mathcal{P}=\tau_{i j} \frac{\partial u_{i}}{\partial x_{j}}
$$

where

$$
\begin{gathered}
\tau_{i j}=\mu_{t}\left(2 S_{i j}-\frac{2}{3} \frac{\partial u_{k}}{\partial x_{k}} \delta_{i j}\right)-\frac{2}{3} \rho k \delta_{i j} \\
S_{i j}=\frac{1}{2}\left(\frac{\partial u_{i}}{\partial x_{j}}+\frac{\partial u_{j}}{\partial x_{i}}\right)
\end{gathered}
$$


The recommended wall boundary conditions are: $k_{\text {wall }}=0, \omega_{\text {wall }}=60 \nu /\left[\beta_{1}\left(\Delta d_{1}\right)^{2}\right]$. A complete description of each of the terms in the standard SST equations can be found in Menter [8].

A common variant of the SST model is termed SST-V, for which the production term makes use of the local magnitude of vorticity $\Omega$ :

$$
\mathcal{P}=\mu_{t} \Omega^{2}-\frac{2}{3} \rho k \delta_{i j} \frac{\partial u_{i}}{\partial x_{j}}
$$

This vorticity source term is often close to the exact source term in boundary layer flows [15], and its use can avoid some numerical difficulties sometimes associated with the use of the exact source term. Other variants of SST include SST-2003 [16] and SST-sust [10]. The main change in SST-2003 is a modification to the definition of $\mu_{t}$, Eq. (5):

$$
\mu_{t}=\rho \frac{a_{1} k}{\max \left(a_{1} \omega, S F_{2}\right)}
$$

where $S=\sqrt{2 S_{i j} S_{i j}}$. The SST-sust model is identical to the original SST model except for the addition of constant sustaining terms $\beta^{*} \omega_{\text {farfield }} k_{\text {far field }}$ and $\beta \omega_{\text {farfield }}^{2}$ in Eqs. (3) and (4), respectively. In the free stream, these have the effect of exactly canceling the destruction terms if $k=k_{\text {farfield }}$ and $\omega=\omega_{\text {farfield }}$, thus preserving free stream levels of $k$ and $\omega$. Inside the boundary layer, the terms are generally orders of magnitude smaller than the destruction terms for reasonable free stream turbulence levels (say, $T u=1 \%$ or less), and therefore have little effect. The main advantage of the use of sustaining terms is that they remove ambiguity associated with far field boundary conditions. For SST (as well as other similar two-equation models), there is free stream decay of $k$ and $\omega$, and local ambient levels can vary depending on distance to the outer boundary. For SST-sust, $k$ and $\omega$ do not decay in the free stream. The recommended far field boundary conditions for SST-sust are: $k_{\text {farfield }}=10^{-6} U_{\infty}^{2}$ and $\omega_{\text {farfield }}=5 U_{\infty} / L$. The far field boundary conditions for SST, SST-V, and SST-2003 tend to vary depending on the code and application. For all results reported here, $k_{\text {farfield }}=9 \times 10^{-9} a_{\infty}^{2}$ and $\omega_{\text {far field }}=1 \times 10^{-6} \rho_{\infty} a_{\infty}^{2} / \mu_{\infty}$.

At the time of this writing, only the SA and SST models and their variants have been posted to the website. As the website database grows, it is planned to eventually include other models, particularly those that see wide use in the aerospace community.

\section{Results}

Sample results are first described for flow over a zero-pressure-gradient (ZPG) flat plate using two different compressible Navier-Stokes CFD codes, CFL3D [17] and FUN3D [18]. The former is a cellcentered structured code and the latter node-centered unstructured. These two codes have both undergone some level of verification using MMS for turbulent flows [4]. The flow conditions are $M=0.2$, $R e=10$ million based on the full length of the plate $(L=2)$, or $R e=5$ million per unit length. Although not shown, when using SA and SST-V both codes yield reasonable results in terms of reproducing wellknown ZPG law-of-the-wall behavior and wall skin friction for which correlations and experimental data exist. This is a matter of validation, which is not the main concern here. Instead, the main concern is verification: what are the expected results for a given model, properly implemented, on a refined grid? If a model has been implemented consistently in two different CFD codes, then both codes should yield the same grid-resolved solution.

For the flat plate, all results were solved using the same series of structured grids (solved both as quadrilateral elements and cut into triangular elements in the unstructured code FUN3D). Grid sizes ranged all the way from the finest $545 \times 385$ to the coarsest $35 \times 25$, and all were members of the same grid family, achieved by removing every other point in each coordinate direction for each coarser grid level. The finest grid had a wall normal spacing of $y=5 \times 10^{-7}$, yielding an average $y^{+}$of approximately 0.1 over the plate. The coarsest grid gave average $y^{+} \approx 1.7$. The grid extended from $x=-0.3333 L$ upstream of the start of the plate to $x=2 L$ at the end of the plate. The upper boundary was located at $y=L$. For this and all other reported results, the CFD codes were converged iteratively such that the density residual was driven well below $10^{-11}$.

Fig. 1(a) and (b) show wall surface skin friction at $x=0.97$ and the integrated drag coefficient, respectively, using the SA model. Results are plotted as a function of $h$, a measure of the average grid spacing. As $h \rightarrow 0$ (indicating finer and finer grid), results from the two codes and results using different grid element types approach each other, demonstrating consistent results as the grid is refined. A similar set of plots is shown in Fig. 2(a) and (b) for the SST-V model. 
Uncertainty estimation procedures are sometimes difficult to use, particularly when (1) CFD results exhibit oscillatory convergence behavior, or (2) when results lie outside of the elusive "asymptotic range" of grid convergence where Richardson extrapolation is valid. Also, in practice CFD can exhibit apparently inconsistent order-property behavior. In other words, although one expects a nominally secondorder code to show $p \approx 2$ (where $p$ is the spatial order of accuracy, and the error is proportional to $h^{p}$ ), in practice the computed range can vary widely (e.g., from less than 1 to 3 or more). It is important to note that as grids are refined, certain characteristics of a scheme such as first-order treatment of boundary conditions, which may not affect the overall order-property behavior for coarser grids, can become the dominant error source. Also, as discussed in Diskin et al.[19], details about the scheme, grid type, grid stretching, and body curvature can all have profound effects on the resulting accuracy.

Using the uncertainty estimation procedure from Celik et al.[20], the finest three grids for the flat plate were used to quantify the grid convergence properties. Results are shown in Table 1 . The computed $p$ on the finest grid level came out to between 0.78 and 1.98 for the properties looked at, with the lowest order tending to occur for the grids cut into triangles. In this table, the fine-grid convergence index (defined in Celik et al.[20]) represents a measure of the fine grid solution error, including a factor of safety. It is important to note here that particular solution quantities using two different CFD codes are not necessarily equal in accuracy, even when using identical grids. Furthermore, the same code on two different grids can converge at different rates. Hence it is necessary to perform an independent grid resolution study for each code and grid combination, to insure grid-independent consistent solutions.

A second case focused on the development of the free planar shear layer following the passing of two different streams over a thin plate (see Fig. 3). The smaller inner stream had Mach number near $M=0.5$, whereas the outer larger stream had a Mach number near $M=0.25$. This can also be considered as a planar co-flowing jet [21]. The Reynolds number was $R e=50,000$ based on the full inner jet width ( $L=1$ here).

For this case only quadrilateral grids have been used to date, ranging from the finest grid with 327,680 grid cells to the coarsest grid with 1280 grid cells. The downstream and upper boundaries were located at $x=200$ and $y=100$, respectively. Typical results are shown in Figs. 4 and 5 . Again, the same turbulence model (SA or SST-V) was shown to approach consistent results for the two codes as the grid was refined. Note that some of the cases exhibited oscillatory convergence behavior for certain properties. If only one code was being used, this behavior would make it difficult to accurately estimate the uncertainty, because an order $p$ cannot be established. For example, Eca and Hoekstra [22] assign an uncertainty of 3 times the maximum difference between all available solutions when oscillatory convergence occurs. However, since both codes here are consistently approaching the same result with grid refinement, the use of two codes in this case can provide a higher confidence estimate of the discretization error levels for a given grid and code. This is a strong argument for using more than one (verified) code when conducting CFD grid convergence studies. For example, in Fig. 5(a), based on the convergence properties of both codes one can confidently assert that the CFL3D fine grid solution is well less than $0.5 \%$ in error from an infinite-grid solution. However, taken alone the oscillatory convergence of CFL3D for this quantity leaves more room for doubt: the method of Eca and Hoekstra (using the finest 3 grids) would estimate fine grid solution error to be greater than $4 \%$.

The final case described here is a $3 \mathrm{D}$ bump in a channel. There is no experiment associated with this case; it is purely a CFD verification exercise, chosen because the wall bump shape is analytic and smooth. There is no flow separation present. Although not discussed here, a 2D verification bump case is also provided on the website. The lower wall is a curved viscous-wall bump extending from $x=0$ to 1.5 at the two sides of the computational domain $y=0$ and $y=-1$, but curved to lie further downstream at $y$ locations in-between. The maximum bump height ( $z$-direction) is 0.05 . The " $2 \mathrm{D}$ " definition of the bump at the $y=0$ plane is:

$$
\begin{aligned}
& z=0.05\left[\sin \left(\frac{\pi x}{0.9}-\frac{\pi}{3.0}\right)\right]^{4} \quad 0.3 \leq x \leq 1.2, \quad y=0 \\
& z=0 \quad 0 \leq x<0.3, \quad 1.2<x \leq 1.5
\end{aligned}
$$

The $x$-location of any position on the bump varies in the spanwise direction between $y=0$ and $y=-1$ according to:

$$
x=x_{0}+0.3(\sin (\pi y))^{4} \quad-1 \leq y<0
$$

where $x_{0}$ is any given $x$-location of the "2D" shape at $y=0$. A sketch of the 3D bump shape is shown in Fig. 6(a). The freestream conditions were $M=0.2$ and $R e=3$ million per unit length. 
All results were solved using the same series of structured grids (only quadrilateral grids have been used to date). Grid sizes ranged from the finest $65 \times 705 \times 321$ (14.7 million grid points) to the coarsest $5 \times 45 \times 21$ (4725 grid points). The finest grid had minimum spacing at the wall of $y=1 \times 10^{-6}$, giving an approximate average $y^{+}$of 0.12 over the plate at the Reynolds number run. The coarsest grid had $y^{+} \approx 2.0$. The grid inflow, outflow, and top boundaries were located at $x=-25, x=26.5$, and $z=5$, respectively.

Only results using SA are shown here. SST-V results using the same grid sequence are similar. Pressure coefficients on the bump surface as well as at $y=0$ are shown on the $33 \times 353 \times 161$ grid in Fig. 6(b) (results are visually essentially identical using both codes on the finer grids). Convergence with grid refinement is shown in Fig. 7 for the bump forces, including lift coefficient, drag coefficient, and pressure and viscous components of drag coefficient. The two codes produced similar trends and approached essentially identical results as the grid was refined, providing further evidence of consistent model implementation.

It should be borne in mind that 3D grid refinement studies that use successive coarsening by removing every other point in each coordinate direction very quickly produce grids that are too coarse to yield adequate solutions. For example, the $17 \times 177 \times 81$ grid (approximately 244,000 grid points) produced drag levels that were nearly $10 \%$ in error from an extrapolated infinite solution, and the $9 \times 89 \times 41$ grid (approximately 33,000 grid points) had error levels near 50\%. In order to obtain 3 or more useful and usable "fine enough" 3D grids, 3D grid studies are sometimes performed by attempting to parametrically employ less than a factor of 2 change in grid spacing in each coordinate direction for successive grids. For complex configurations, however, it can be difficult to ensure that the grids produced in this way are of the same family.

\section{Conclusions}

A turbulence model verification effort was described, which is part of a larger goal of an AIAA discussion group to help improve the consistency, verification, and validation of turbulence models within the aerospace community. A NASA website - http://turbmodels.larc.nasa.gov - has been established to provide the CFD community with a resource for (1) finding turbulence models, and (2) verifying their own coding of turbulence models. This latter capability is made possible by providing simple test cases and grids, along with sample results (including grid convergence studies) from one or more previouslyverified codes. Furthermore, by listing accepted versions of the turbulence models as well as published variants, this website establishes naming conventions in order to help avoid confusion when comparing results from different codes.

This paper described some of the turbulence model variations that exist for the popular SpalartAllmaras and Menter shear-stress-transport models, and used two specific versions of these models in two independent computer codes to demonstrate consistency with grid refinement for several cases. Combining consistency in model implementation in two or more codes along with thorough grid convergence studies for a range of problems is a powerful methodology that can help to establish the grid-resolved solutions and the uncertainties on finite grids. This can be very useful for the common situation in which traditional uncertainty methods (such as those involving Richardson extrapolation) fail for a single code because of oscillatory convergence or inability to achieve the asymptotic range of grid convergence.

\section{References}

[1] Vassberg, J. C., Tinoco, E. N., Mani, M., Levy, D., Zickuhr, T., Mavriplis, D. J., Wahls, R .A., Morrison, J. H., Brodersen, O. P., Eisfeld, B., and Murayama, M., "Comparison of NTF Experimental Data with CFD Predictions from the Third AIAA CFD Drag Prediction Workshop," AIAA Paper 20086918, August 2008.

[2] Viti, V., Huang, G., and Bradshaw, P., "Numerical Study of Stress-Transport Turbulence Models: Implementation and Validation Issues," Computers \& Fluids, Vol. 37, 2007, pp. 1373-1383.

[3] Eca, L., Hoekstra, M., Hay, A., and Pelletier, D., "A Manufactured Solution for a Two-Dimensional Steady Wall-Bounded Incompressible Turbulent Flow," International Journal of Computational Fluid Dynamics, Vol. 21, Nos. 3-4, 2007, pp. 175-188. 
[4] Rumsey, C. L. and Thomas, J. L., "Application of FUN3D and CFL3D to the Third Workshop on CFD Uncertainty Analysis," NASATM-2008-215537, November 2008.

[5] Carlson, J. R., Duquesne, N., Rumsey, C. L., and Gatski, T. B., "Computation of Turbulent Wake Flows in Variable Pressure Gradient," Computers \& Fluids, Vol. 30, 2001, pp. 161-187.

[6] Rubinstein, R., Rumsey, C. L., Salas, M. D., and Thomas, J. L., "Turbulence Modeling Workshop," NASA/CR-2001-210841, March 2001; also ICASE Interim Report No. 37, 2001.

[7] Spalart, P. R., and Allmaras, S. R., "A One-Equation Turbulence Model for Aerodynamic Flows," Recherche Aerospatiale, No. 1, 1994, pp. 5-21.

[8] Menter, F. R., "Two-Equation Eddy-Viscosity Turbulence Models for Engineering Applications," AIAA Journal, Vol. 32, No. 8, 1994, pp. 1598-1605.

[9] Spalart, P. R., "Trends in Turbulence Treatments," AIAA Paper 2000-2306, June 2000.

[10] Spalart, P. R. and Rumsey, C. L., "Effective Inflow Conditions for Turbulence Models in Aerodynamic Calculations," AIAA Journal, Vol. 45, No. 10, 2007, pp. 2544-2553.

[11] Shur, M. L., Strelets, M. K., Travin, A. K., Spalart, P. R., "Turbulence Modeling in Rotating and Curved Channels: Assessing the Spalart-Shur Correction," AIAA Journal, Vol. 38, No. 5, 2000, pp. 784-792.

[12] Catris, S. and Aupoix, B., "Density Corrections for Turbulence Models," Aerospace Science and Technology, Vol. 4, 2000, pp. 1-11.

[13] Edwards, J. R. and Chandra, S. "Comparison of Eddy Viscosity-Transport Turbulence Models for Three-Dimensional, Shock-Separated Flowfields," AlAA Journal, Vol. 34, No. 4, 1996, pp. 756-763.

[14] Rung, T., Bunge, U., Schatz, M., and Thiele, F., "Restatement of the Spalart-Allmaras EddyViscosity Model in Strain-Adaptive Formulation," AIAA Journal, Vol. 41, No. 7, 2003, pp. 1396-1399.

[15] Menter, F. R., "Improved Two-Equation k-omega Turbulence Models for Aerodynamic Flows," NASA TM 103975, October 1992.

[16] Menter, F. R., Kuntz, M., and Langtry, R., "Ten Years of Industrial Experience with the SST Turbulence Model," Turbulence, Heat and Mass Transfer 4, ed: K. Hanjalic, Y. Nagano, and M. Tummers, Begell House, Inc., 2003, pp. 625 - 632.

[17] Krist, S. L., Biedron, R. T., and Rumsey, C. L., "CFL3D User's Manual (Version 5.0)," NASA TM1998-208444, June 1998.

[18] Nielsen, E. J. and Anderson, W. K., "Recent Improvements in Aerodynamic Design Optimization On Unstructured Meshes", AIAA Journal, Vol.40, No. 6, 2002, pp. 1155-1163.

[19] Diskin, B., Thomas, J. L., Nielsen, E. J., Nishikawa, H., and White, J. A., "Comparison of NodeCentered and Cell-Centered Unstructured Finite-Volume Discretizations. Part I: Viscous Fluxes," AIAA Paper 2009-597, January 2009.

[20] Celik, I. B., Ghia, U., Roache, P. J., Freitas, C. J., Coleman, H., and Raad, P. E., "Procedure for Estimation and Reporting of Uncertainty Due to Discretization in CFD Applications," Journal of Fluids Engineering, Vol. 130, July 2008, 078001.

[21] Rajaratnam, N., Developments in Water Science: Turbulent Jets, Elsevier Scientific Publishing Company, New York, 1976.

[22] Eca, L. and Hoekstra, M., "Evaluation of Numerical Error Estimation Based on Grid Refinement Studies With the Method of Manufactured Solutions," Computers \& Fluids, Vol. 38, 2009, pp. 15801591. 
Table 1. Computed convergence behavior for flat plate case

\begin{tabular}{llcc}
\hline Property & Code & Computed apparent order, $p$ & Fine-grid convergence index, \% \\
\hline$C_{f}$ at $x=0.97$ & CFL3D, SA & 1.98 & 0.017 \\
& FUN3D, SA, quad & 1.34 & 0.028 \\
& FUN3D, SA, tri & 0.85 & 0.592 \\
CFL3D, SST & 1.21 & 0.277 \\
& FUN3D, SST, quad & 1.39 & 0.256 \\
& FUN3D, SST, tri & 1.24 & 0.300 \\
\hline$C_{D}$ & GFL3D, SA & 1.75 & 0.027 \\
& FUN3D, SA, quad & 0.80 & 0.269 \\
FUN3D, SA, tri & 0.78 & 0.854 \\
& GFL3D, SST & 1.34 & 0.222 \\
& FUN3D, SST, quad & 1.07 & 0.914 \\
& FUN3D, SST, tri & 0.99 & 0.657 \\
\hline
\end{tabular}
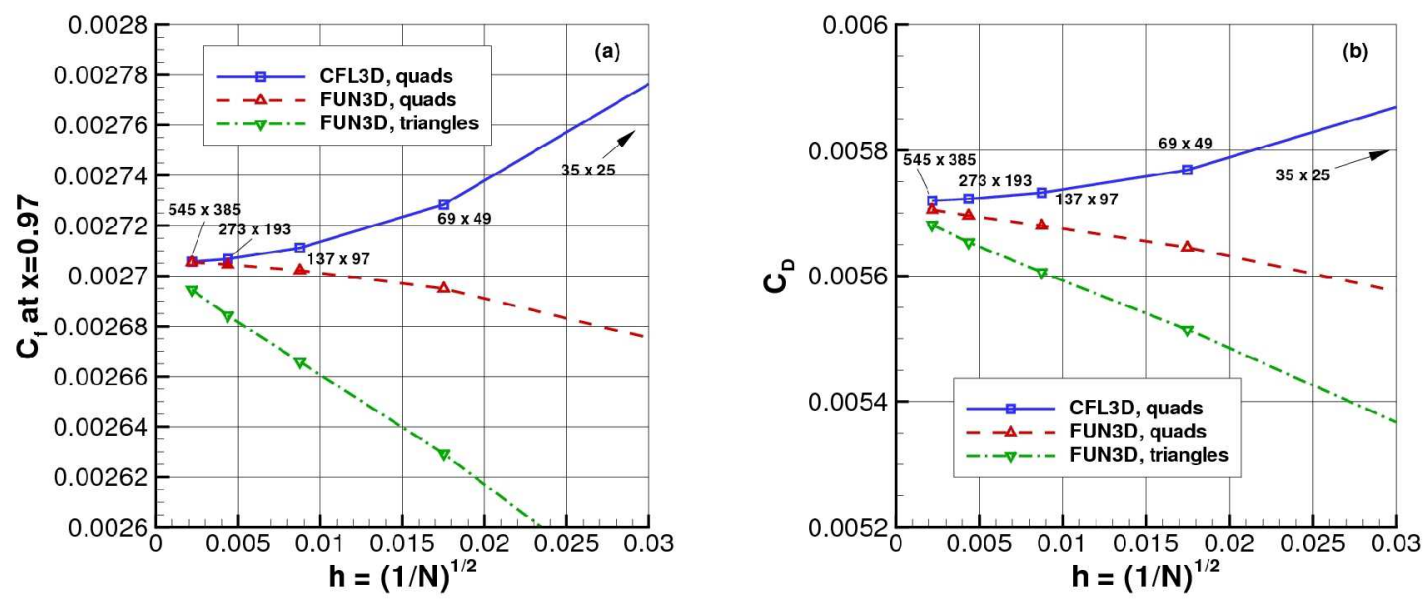

Figure 1. Results for flat plate using two independent codes with the SA model; (a) skin friction coefficient at $x=0.97$, (b) drag coefficient.
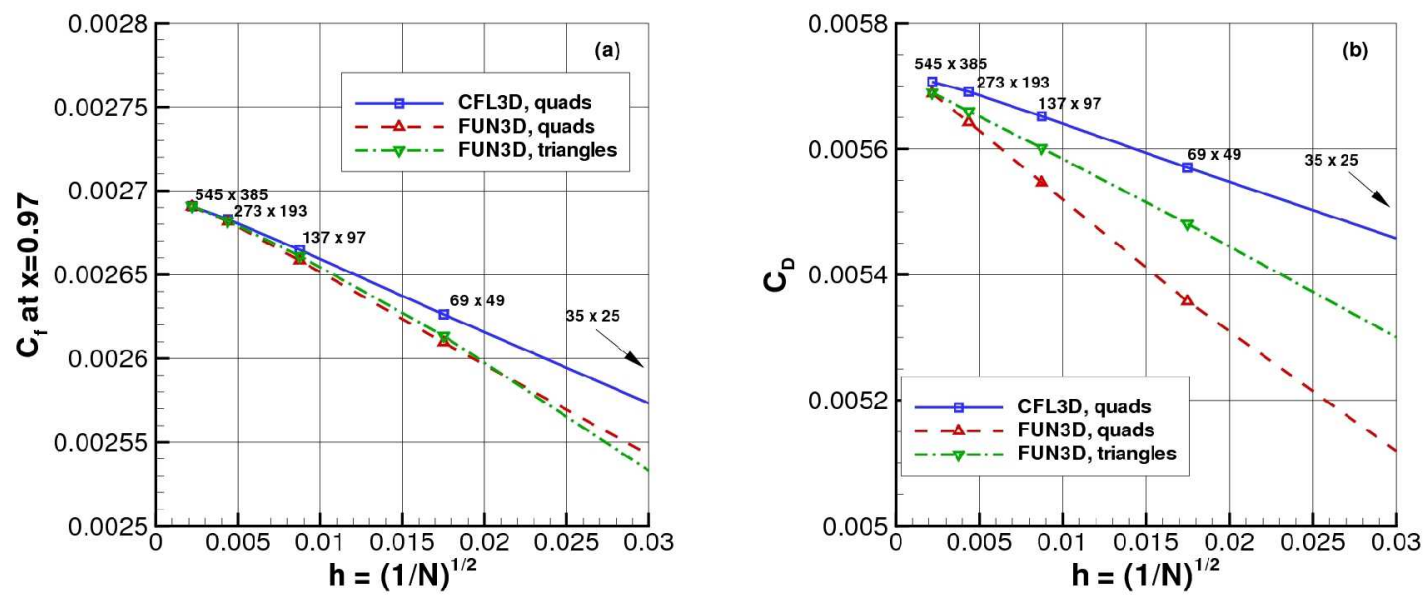

Figure 2. Results for flat plate using two independent codes with the SST-V model; (a) skin friction coefficient at $x=0.97$, (b) drag coefficient. 


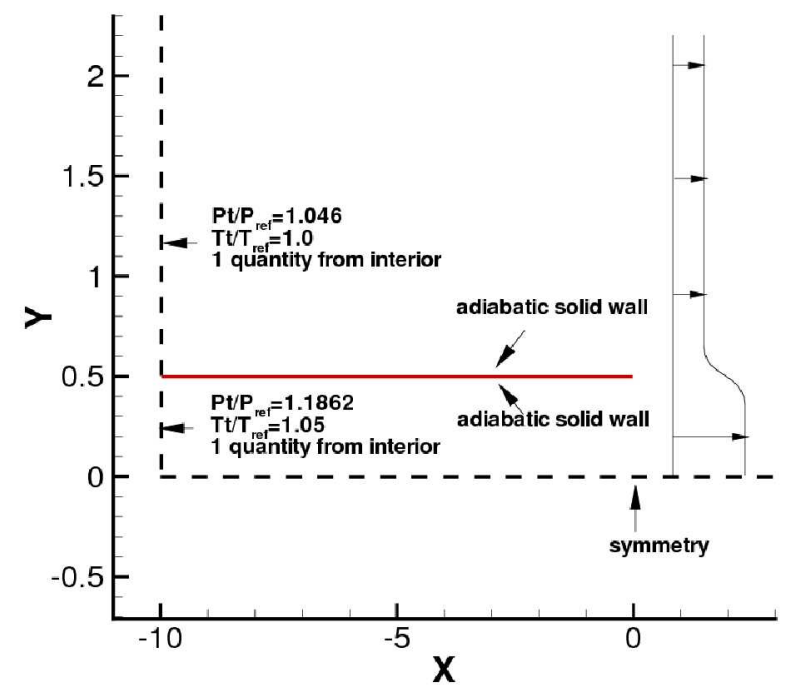

Figure 3. Description of planar shear case.
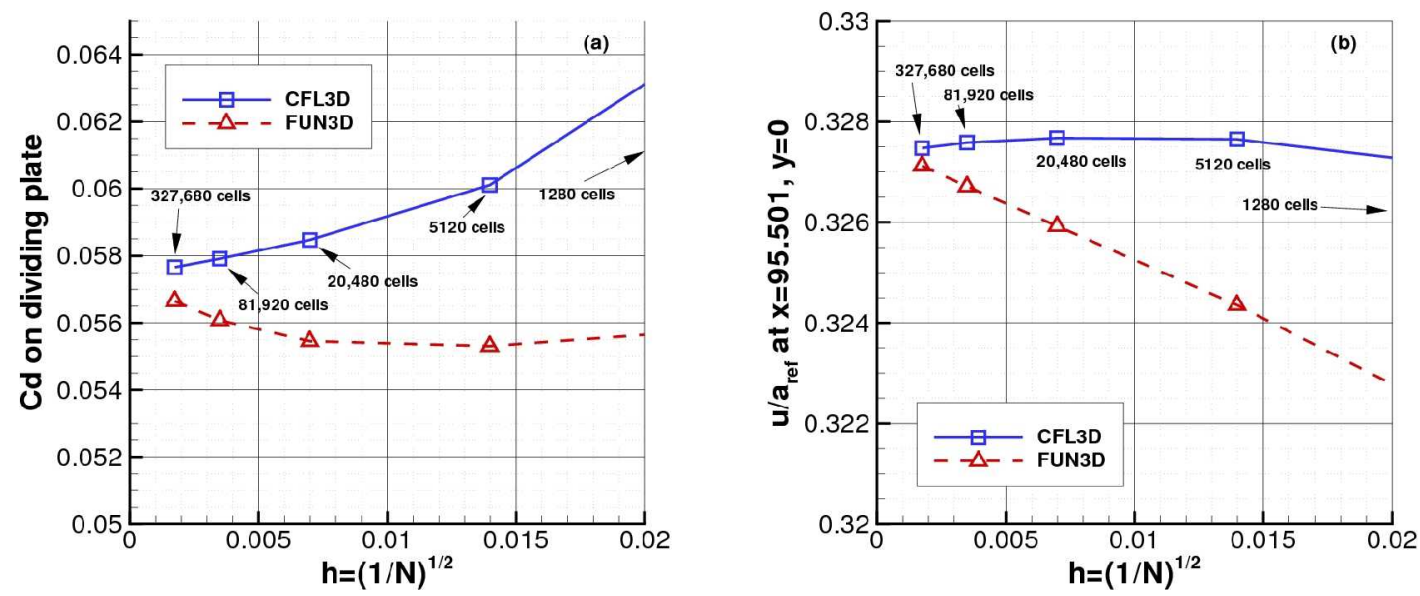

Figure 4. Results for planar shear using two independent codes on a quadrilateral grid with the SA model; (a) drag coefficient on thin plate, (b) $u / a_{\text {ref }}$ along the centerline at $x=95.5$.
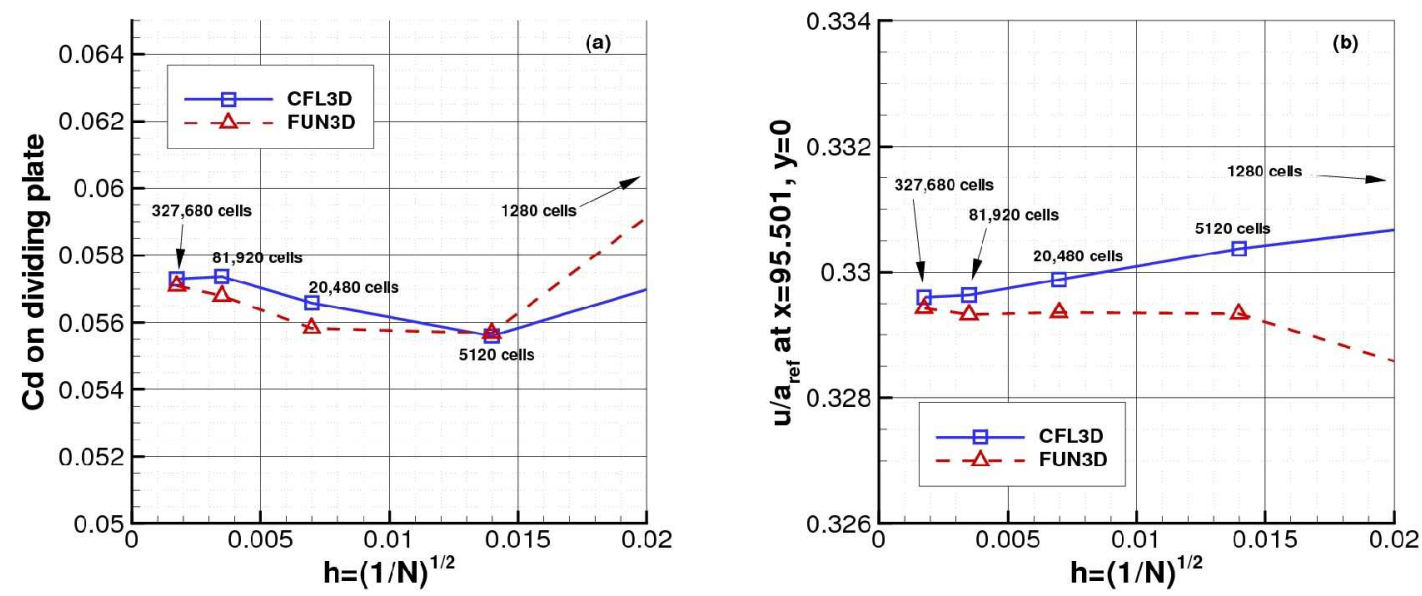

Figure 5. Results for planar shear using two independent codes on a quadrilateral grid with the SST-V model; (a) drag coefficient on thin plate, (b) $u / a_{\text {ref }}$ along the centerline at $x=95.5$. 

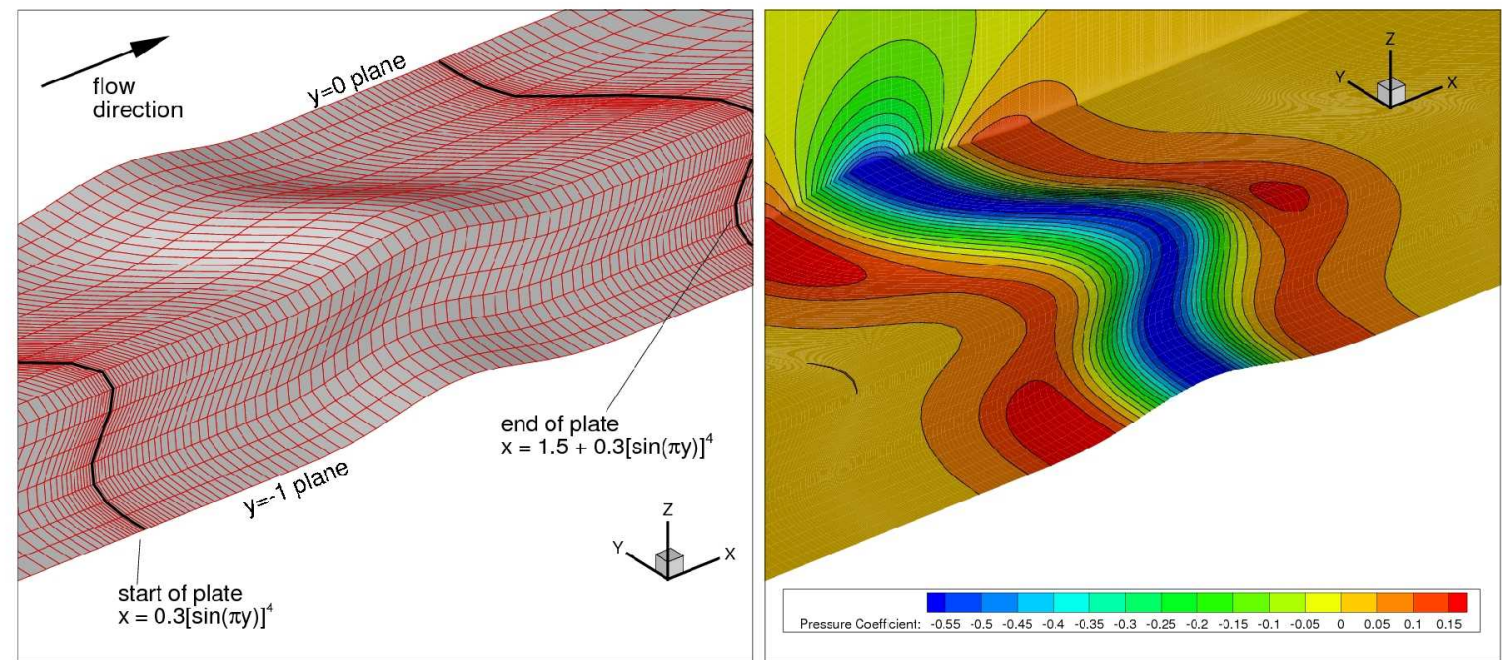

Figure 6. Overview of 3D bump test case; (a) sketch of bump shape, (b) pressure coefficients using SA model on $33 \times 353 \times 161$ grid.
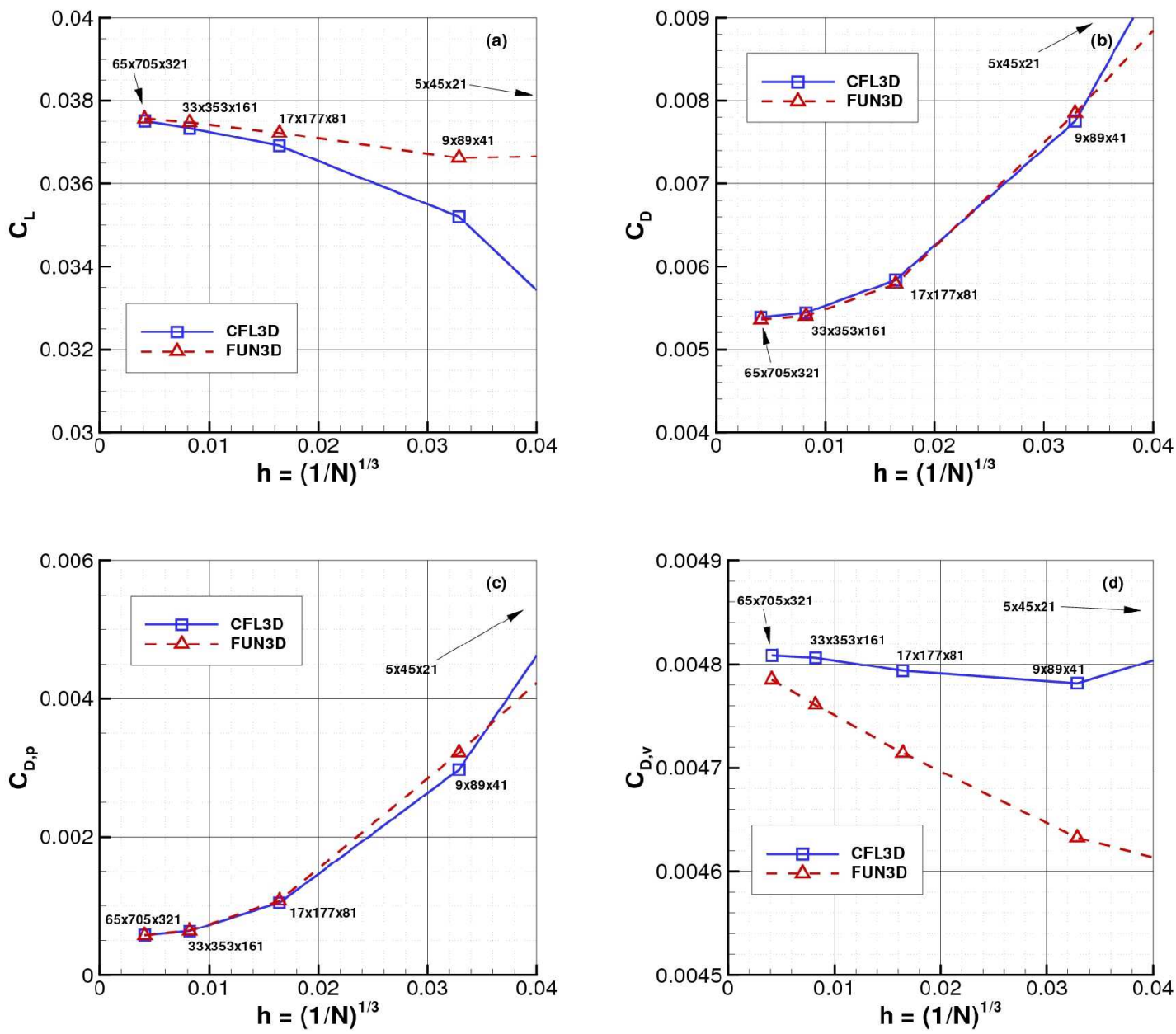

Figure 7. Results for 3D bump using two independent codes with the SA model; (a) $C_{L}$, (b) $C_{D}$, (c) $C_{D, p}$, (d) $C_{D, v}$. 